\title{
Domain and Specification Models for Software Engineering
}

\author{
Neil Iscoe, Zheng-Yang Liu, Guohui Feng \\ EDS Research, Austin Laboratory \\ 1601 Rio Grande, Ste 500 \\ Austin, Texas 78701 \\ iscoe@austin.eds.com
}

\begin{abstract}
This paper discusses our approach to representing application domain knowledge for specific software engineering tasks. Application domain knowledge is embodied in a domain model. Domain models are used to assist in the creation of specification models. Although many different specification models can be created from any particular domain model, each specification model is consistent and correct with respect to the domain model. One aspect of the system-hierarchical organization is described in detail.
\end{abstract}

\section{Introduction}

Creating, maintaining and evolving software systems requires an understanding of both programming knowledge and application domain knowledge. Programming knowledge is relatively well understood. It is formal, modeled in a variety of ways, explicit enough to be taught to novices, and general enough to apply across many domains. Although empirical field studies (Curtis, et al., 1988) have shown that application domain knowledge is critical to the success of large projects, this knowledge is rarely modeled as needed. It is usually implicitly embodied in the application code rather than explicitly recorded and maintained separately from the code. Even when the knowledge is recorded, it is generally stored in voluminous natural language documents in an informal rather than a formal manner. Although problem-specific languages partially remedy this situation, they still capture domain knowledge in an ad hoc rather than a systematic manner. Furthermore, these languages are generally not designed in such a way that the results can be generalized or even replicated.

Application domain models are representations of relevant aspects of application domains that can be used for different operational goals in support of specific software engineering tasks or processes. Domain models determine what there is in the world for reasoning about given application domains and sanction the types of inferences allowed.

Operational goals are always implicit in the construction of a domain model and are essential to understanding the form and content of that model. Unlike generalized knowledge representation projects such as Cyc (Lenat, 1990) that attempt to provide a basis for modeling encyclopedic knowledge, domain modeling explicitly acknowledges the commonly held view (Amarel, 1968) that representations are designed for particular purposes. These purposes-the operational goals-inherently bias any particular solution and dictate the final form of the model. As real-world domains are infinitely rich and diverse, we inevitably adopt particular perspectives in deciding what is relevant with respect to given tasks when formulating models (Liu and Farle $;$, 1991). Even within the field of domain modeling, miny different operational goals and modeling projects are being pursued (Iscoe, et al. 1991).

In the next section, we give an overview of the domain modeling research at EDS and our corresponding operational goals. We then introduce a model reformulation concept-the generation of multiple specification models from a single domain model. The remainder of the paper focuses on one of the mechanisms which allows a specification designer to rapidly construct specification models that are consistent and correct with respect to the original domain model.

\section{Domain Modeling Research}

EDS specializes in creating software for a variety of industries. Each industry area such as utilities, finance, or health insurance has an associated body of knowledge which is critical to the understanding of specification and implementation of software systems. Domain expertise is acquired by personnel over a period of years, and the company is organized into strategic business units (SBUs) so that knowledge about a particular industry can be maintained over time.

At the EDS Austin research laboratory, we are attempting to create a domain modeling system which can achieve the following operational goals:

- Requirements \& Specifications-Eliciting, verifying, and formalizing software requirements and specifications,

- Program Transformation/Generation-Transforming a specification into efficient executable code,

- Reverse Engineering-Identifying the semantics of existing code in terms of a partial specification,

- Explanation, Education \& Communication-Capturing and communicating application domain knowledge.

The realization of these operational goals is consistent with our long-term plan for creating knowledge-based tools to support programming-in-the-large (Barstow, 1988) development. The domain modeling approach provides ample opportunities for investigating and creating new development paradigms. 




Figure 1. Domain Modeling with Operational Goals

Figure 1 illustrates the context in which we model. The industry knowledge for each SBU is instantiated into a domain model, which then serves as a source of knowledge for programs (the ovals) to achieve our operational goals. In the figure, the specification model (rectangle) contains the specification for a specific system within an application domain. Because one of our goals is to generate executable code, we require that any particular specification model be consistent. A very large but finite number of specification models can be created which are consistent and are correct with respect to a particular domain model.

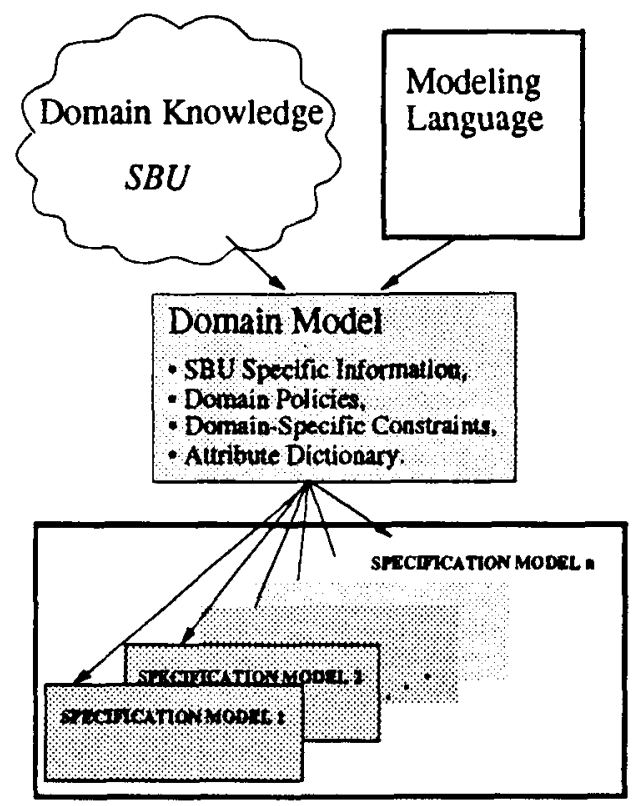

Figure 2. Instantiating Specification Models
Figure 2 illustrates the two separate modeling tasks required by our approach. Domain experts interact with a system to store their knowledge in terms of a domain model. Specification designers then use the system to build specification models which satisfy constraints in the domain model.

In order to create a specification model, the designer selects a set of relevant policies and constraints from the domain model that must be included and enforced in the specification model. The constraints include intra-attribute as well as inter-attribute relationships within and across entities relevant to the task at hand.

\section{Dynamic Knowledge Structure}

The remainder of this paper presents one aspect of our meta-model representation that is relevant to this workshop-dynamic restructuring of a hierarchically organized domain knowledge.

While most would agree that hierarchical organizational strategies provide a reasonable way to structure knowledge within complex domains, the creation of a hierarchical structure, like any type of representational scheme, imposes a particular view of the world. Unfortunately, there is no particular view that is optimal for every application. Although the programs within a particular application share the same legal, physical, and economic constraints, the construction of any particular specification model depends upon a set of policy decisions that determine how cases are handled. Furthermore, software in the large systems are continually changing in such a manner that the concept of a static hierarchy is insufficient to capture the process of system evolution.

Consider software systems that manage the payment of health insurance claims. Although conceptually simple, these systems handle hundreds of thousands of different 
cases. One way to represent these cases is to enumerate the leaf nodes of the hierarchies created by the appropriate partitioning of attributes such as gender, age, family_status, previous_condition, employment, deductibles, copayments, prognosis, and so on. Unfortunately, the tree structure created by case expansion not only obscures relevant and interesting cases, but is also a monolithic structure. It is a paradox of object-oriented approaches that well-adapted structures are not adaptable to new situations.

Because of the combinatorial explosion of the leaf nodes, it makes sense to handle the cases at as high a level as possible. Term subsumption systems such as CLASSIC (Borgida, et al. 1989) automate this process by determining the place in a hierarchy in which terms are subsumed. But subsumption systems assume a single structure in which all sub-models can belong. In the case of applications such as health insurance, individual modules may have different hierarchical structures and still maintain the integrity and constraint rules of the domain model.

\section{Attribute Definitions}

Attributes are normally considered as data values or slot fillers within a class or frame. However, the standard treatment of attributes as lists of data values with some underlying machine representation fails both to capture sufficient semantic information from the application domain and to state definitions with sufficient formality to allow semantics-related consistency checks.

Attributes are functions which define how a set of objects is mapped within a class. One type of attribute has a value set represented by a nominal scale which consists of a set of values, $\mathcal{U}(\mathrm{A})=\left\{\mathrm{C}_{1}, \ldots \mathrm{C}_{\mathrm{n}}\right\}$.

The semantics of an application domain are maintained by creating categories in such a way that items to be categorized with respect to a particular attribute are as homogeneous as possible within a category and as heterogeneous as possible between categories. Examples of nominal scales abound and map cleanly to the notion of enumerated type as shown below:

(Colors

$\begin{array}{ll}\text { :type } & \text { nominal_scale } \\ \text { :values } & \text { (Red Yellow Green Blue) }\end{array}$

The next type of attribute is an ordinal scale-a nominal scale in which a total ordering exists among the categories. Interval and ratio scales are the more quantitative scales and add definitions of dimensions, units, and granularity.

This brief description of attribute type was included to allow the reader to understand the examples in this paper. Attributes have additional types and a number of other properties which are explained in (Iscoe, et al 1992).

\section{Hierarchical Decomposition}

Hierarchies are a natural way to view and organize information and, at some level of abstraction, are a mart of most object-oriented and knowledge represe $i$, tion languages. Unfortunately, the simplicity of these ci - nts can sometimes obscure the semantics that a mo.t is attempting to capture. That one's needs dictate one's ontological choice is a fundamental premise of knowledge engineering. The ability to systematically define a new set of attributes by partitioning the value sets of old attributes and then using these new attributes to reclassify the domain in accordance with the new requirements is a fundamental aspect of our attribute characterization. By preserving the "ontological map" as a component of the attribute, the domain modeler can shift between the differing paradigms modeled by various classes of objects.

Attribute characterization provides a representation and systematic methodology for the partitioning of attributes that facilitates the way they are organized, subdivided, and built into hierarchies. An attribute restriction is a new attribute whose value set and set of applicable relations are subsets of the original attribute.

Creating a new attribute serves the dual purpose of creating a set of views on the old aturibute as well as creating a new attribute. Often, new attributes are defined in terms of old attributes by partitioning the original value set and then equating each new attribute value with an element of the partition. As an example, an accounts receivable (AR) system may use the attribute days_to_payment whose value is the average number of days it takes for the client to pay a bill.

$\begin{array}{ll}\text { (days_to_payment: } \\ \text { :type } & \text { ratio_scale } \\ \text { :dimension } & \text { time } \\ \text { :unit } & \text { days } \\ \text { :min } & 0 \\ \text { :max } & 360 \text { ) }\end{array}$

From the standpoint of AR applications, a more useful attribute might be :

(type_of_payer.

:type Ordinal_scale

:Ordered_by lateness of paymen

:values (pays_on_time slow_pay dead_beat))

This new aturibute will be defined by partitioning the value set of days_to_payment, Vp by subdividing the range of values, then equating each value with one of the elements of the partition as illustrated in figure 3 and described as follows:

(type_of_payer

:mapped_from days_to_payment

(pays_on_time $\quad(<=30)$

(slow_pay

(AND $(>30)(<90)))$

(dead_beat $(>=90))))$

days_to_payment:

Ratio_scale Time in Days (Min 0) (Max 360)



Figure 3-Partitioning days_to_payment 
Note that the days_to_payment attribute is based on a ratio scale while the type_of_payer attribute is based on an ordinal scale. In general a defined attribute represents a loss of information (in this example, the number of days overdue) in return for a more useful and inherently less detailed category.

\section{Using Population Parameters}

Population parameters facilitate the formation of new attributes. For example, some graduate admissions committees use interval-scaled GRE scores to separate applicants into acceptance categories. Population parameters allow designers to create new attributes based on restrictions to the original attribute as shown below:

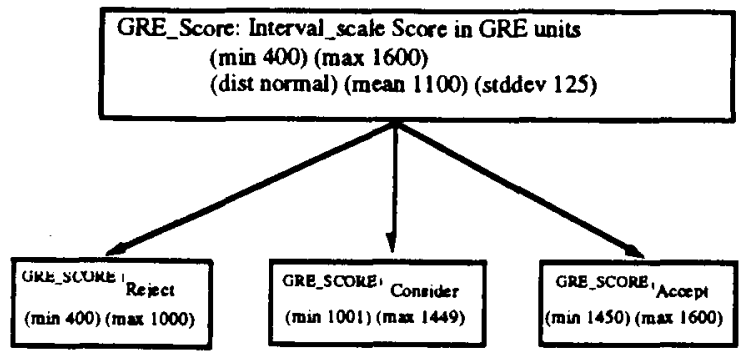

\section{Figure 4 - Using Population Parameters to Restrict an Attribute}

Figure 4 shows the GRE score as an attribute which could be attached to a student. Understanding the distribution of values within the value set of GRE scores allows application designers to create partitions in any one of a variety of ways. For example, assume that an application designer wanted to create an initial partition based on the requirement "accept all students who score in the top $x \%$ on the GRE, consider those who score between $x \%$ and $y \%$, and reject those who score in the bottom $y \%$." Given this type of requirement, the domain model contains the appropriate information to use and an algorithm to produce the correct raw score numbers to achieve such a partition.

Another way that these requirements are sometimes stated is to build a partition based on an absolute raw score. For example, a requirement like "accept all students who score above 1450 on the GRE" can be easily incorporated. Furthermore, this type of specification can be used interactively so that the designer can juggle between raw scores and percentiles until the partitions appropriate for the application domain are produced.

\section{Domain and Specification Models}

In this section we focus on relations between attributes within a single domain model class. For the purposes of this discussion we define the following attributes:
(name
:type identifier)
(eye_color :type nominal_scale
:values (brown, blue, green))
(Gender
:type nominal_scale



Although other constraints exist, domain model classes can be regarded as consisting of sets of attributes which are either required or might be included within a particular domain model. These constraints are expressed as follows:

must_have $(c, a, c o n d)$ - attribute $a$ must be used in class $c$ in a model if condition cond evaluates to true. applicable (c, $a$, cond) - attribute $a$ can be used in class $c$ a model if condition cond evaluates to true.

Within any particular specification model, an attribute is simply classified as used within a class. used $(m, c, a$, cond $)$ - within model $\mathrm{m}$, attribute $a$ is used in class $c$ in model $m$ if condition cond evaluates to true.

The most straight-forward relationship between a domain model and a specification model is that must_have attributes are used in all specification models and applicable attributes are selected by the specification designer.

must_have(c, a, cond) $\leftrightarrow \forall \mathrm{m}$ used(m, c, a, cond)

applicable(c, a, cond) $\leftrightarrow \exists \mathrm{m}$ used(m, c, a, cond) thus

must_have(c,a, cond) $\rightarrow$ applicable(c,a, cond)

For example, in a domain model, name might be required for all specification models, while eye_color could be selected only if it were appropriate for the particular specification model.



The application of these constraints when cond is vacuously true is fairly standard feature in most modeling languages of this type. However, name and eye_color are attributes which are total and are not as interesting as the cases that occur when the attributes are partial functions.

\section{Conditions for Function Evaluation}

Recalling that an attribute is a function which maps objects to a particular property, cond can be interpreted as the condition which must be satisfied for the attribute to be a total instead of a partial function. In other words, cond defines the subset which is the domain of applicability of 
the partial function. For example for a person class hysterectomy is only applicable if the gender is female.

(applicable person Hysterectomy (= Gender female))

The domain modeling system is designed so that the conditions required to establish the proper domain for an attribute are automatically maintained. These conditions are constrained in such a way that tractability is maintained and are of the form $\left(\left(p_{1} a_{1} v_{1}\right) 1 \ldots\left(p_{n} a_{n} v_{n}\right)\right)$, where $p_{i}$ is the name of a predicate, $a_{i}$ is the name of an attribute, and $v_{i}$ is a value of the attribute.

When conditions exish, the following axiom is needed:

(applicable $c$ a cond1) $\rightarrow$

[(used $\mathrm{m} \mathrm{c}$ a cond 2 ) $\rightarrow$ (cond $1 \rightarrow$ cond 2$)$ ]

A user can create a specification model with any particular class hierarchy as long as the domain policies and constraints are satisfied.

Domain and specification model consistency is maintained by a specialized theorem prover. The theorem prover, $S T R+V E$, is an upgraded version of the prover presented in (Bledsoe 1980) for proofs of theorems in general inequalities. A TMS is being constructed to interface between the modeling system and the theorem prover

We are currently experimenting with ways to capture and verify domain modeling constraints by presenting redundant information in a variety of ways. We believe that many of the specification problems in large systems are created when value set changes cause a single case to be changed but fail to correct cases that were identified from a previous inference.

For example, if we assume that hysterectomy is applicable to females, the system can infer that hysterectomy cannot apply to males by using axiom 1, the definition of applicable, and the definition of gender to derive a contradiction.

applicable(c, a, cond) $\leftrightarrow \exists \mathrm{m}$ used(m, c, a, cond)

applicable(P, hys, $[(=$ gender $m)])$

$\neg(=$ Gender, $M) \rightarrow(=$ Gender, $F)$

(= Gender, $M) \rightarrow \neg$ (= Gender, F)

A key point is that when people are presented with value sets they automatically and unconsciously perform substitutions such as the ones listed above. This is a reasonable way to build a model until a value set changes. In large systems, value sets are frequently changed. Consequently, conclusions that were drawn by using negation to infer values become invalid. We use the applicability of conditions and the system's knowledge of value sets to attempt to provide the proper cases for the domain modeler to check when condtions change.

\section{Discussion}

In this paper, we have presented the concept of modeling application domains in order to achieve the operational goals of program specification, code generation, and reverse engineering. The main concept is that multiple specification models can be created that are consistent and "correct" with respect to a domain model. Domain models represent information about a particular industry area. Specification models represent information about a particular system.

Domain and specification models are constructed by using a graphical interface to interactively create a set of rules based on attribute value set partitions and the preceding axioms. The system is being implemented using Motif GUI on SPARC workstations. Although it is currently operating in a single user mode, it is being designed to be accessed simultaneously by multiple domain modelers. We are also trying to accelerate the knowledge capture process by reverse engineering data models that bave been captured by an existing EDS case tool and instantiating them into the appropriate domain models.

\section{References}

Amarel, S. 1968. "On Representations of Problems of Reasoning About Actions," in Machine Intelligence 3, D. Mi nie. Ed., American Elsevier, New York pp. 131-171.

:stow, D. 1985. "Domain-Specific Automatic Prramming," IEEE Transactions on Software Engineering, vol. SE-11, no. 11, pp. 1321-1336.

Barstow, D. 1988. "Artificial Intelligence and Software Engineering," in Shrobe, H., ed., Exploring Artificial Intelligence. AAAI. Morgan Kaufmann, San Mateo, CA.

Bledsoe, W. W., and Hines, L. M. 1980. "Variable Elimination and Chaining in a Resolution-Base Prover for Inequalities," Proceedings of the 5th Conference on Automated Deduction, Les Arcs, France, Springer-Verlag, pp. 70-87.

Borgida, A., Brachman, R.J., McGuinness, D.L., and Resnick, L.A. 1989. "CLASSIC: A structural data model for objects," in Proceedings of the 1989 ACM SIGMOD International Conference on Management of Data, pp. 5967.

Curtis, B., Krasner, H. and Iscoe, N. 1988. "A Field Study of the Software Design Process for Large Systems," Communications of the $A C M$, vol. 31 , no. 11 , pp. 12681287.

Davis, R. 1991. "Knowledge Representation: Broadening the Perspective," AAAI-91 Panel, Anaheim, CA.

Iscoe, N, Browne. J.C., Werth' J. , and Liu, Z.Y, 1992 "Attributes - Building Blocks for Modeling Application Domains," Submitted to IEEE TSE

Iscoe, N., Williams, G. and Arango, G., Eds. 1991. Domain Modeling for Software Engineering, Proceedings of Domain-Modeling Workshop, Austin, Texas.

Lenat, D.B. , Guha, R.V., Pittman, K., Pratt, D., and Shepherd, M. 1990. "Cyc: Toward Programs with Common Sense," CACM, vol. 33, no. 8, pp. $30-49$.

Liu, Z.-Y. and Farley, A. 1991. "Tasks, Models, Perspectives, Dimensions," The 5th International Workshop on Qualitative Reasoning Austin, T:xas, pp. 1 12. 